Working Paper/Document de travail

2007-25

\title{
Managing Adverse Dependence for Portfolios of Collateral in Financial Infrastructures
}

by Alejandro García and Ramazan Gençay 
Bank of Canada Working Paper 2007-25

April 2007

\title{
Managing Adverse Dependence for Portfolios of Collateral in Financial Infrastructures
}

\author{
by \\ Alejandro García ${ }^{1}$ and Ramazan Gençay ${ }^{2}$ \\ ${ }^{1}$ Monetary and Financial Analysis Department \\ Bank of Canada \\ Ottawa, Ontario, Canada K1A 0G9 \\ agarcia@bankofcanada.ca \\ ${ }^{2}$ Department of Economics \\ Simon Fraser University \\ Burnaby, British Columbia, Canada V5A 1S6 \\ rgencay@sfu.ca
}

Bank of Canada working papers are theoretical or empirical works-in-progress on subjects in economics and finance. The views expressed in this paper are those of the authors.

No responsibility for them should be attributed to the Bank of Canada. 


\section{Acknowledgements}

We thank Carol Ann Northcott, Nikil Chande, Dinah Maclean, Greg Tkacz, and Walter Engert for their comments and suggestions on this paper. We also would like to thank Katerine Martin, Devin Ball and Kelly West for their expert technical assistance. We gratefully acknowledge the financial support from the Natural Sciences and Engineering Research Council of Canada, the Social Sciences and Humanities Research Council of Canada, and the Bank of Canada. We thank the Canadian Depository for Securities for providing us with the price data for the equities used in this paper. This paper is part of a two year research program. The first paper of this research program corresponds to Garcia and Gençay (2006). 


\begin{abstract}
We propose a framework that allows a portfolio manager to quantify the probability of simultaneous losses in multiple assets of a collateral portfolio. Using this framework, we propose a methodology to conduct stress tests on the market value of the portfolio of collateral when undesirable extreme dependence occurs. This framework permits us to quantify the potential impact on the portfolio returns of systemic events that change, or 'break down', the historical comovement structure, imposing an adverse extreme dependence. We illustrate our framework using securities pledged as collateral in the Canadian securities clearing and settlement system.

JEL classification: G00, G10, C10

Bank classification: Econometric and statistical methods; Financial markets; Financial stability
\end{abstract}

\title{
Résumé
}

Les auteurs proposent un cadre à l'aide duquel un gestionnaire de portefeuille peut quantifier la probabilité de pertes simultanées sur les actifs d'un portefeuille composé de garanties. La méthodologie qu'ils présentent permet de simuler l'évolution de la valeur de marché d'un portefeuille de garanties dans un scénario de crise caractérisé par une dépendance de queue non souhaitable. Elle leur permet ainsi de quantifier l'incidence potentielle, sur les rendements du portefeuille, de chocs systémiques qui modifient ou rompent la relation historique de covariation et se soldent par une dépendance de queue non souhaitable. Pour illustrer leur méthodologie, les auteurs étudient le cas des titres pouvant servir de garantie au sein du système canadien de compensation et de règlement des opérations sur titres.

Classification JEL : G00, G10, C10

Classification de la Banque : Méthodes économétriques et statistiques; Marchés financiers; Stabilité financière 


\section{Introduction}

Regulators and banking professionals have undertaken several initiatives designed to improve the safety of the global financial system. These initiatives include the development of core principals for systemically important payment systems (BIS 2001a), recommendations for securities settlement systems (BIS 2001b), and recommendations for central counterparties (BIS 2004). These initiatives share a common approach; financial risks, such as market risk, credit risk, etc., are managed by pledging securities as collateral. Collateral acts as a form of insurance, that is, when funds are needed to cover losses, collateral is liquidated to obtain the necessary funds. However, collateral itself may consist of risky assets and thus can change in value over time. An important concern, therefore, is that sufficient collateral is pledged, so that if a failure occurs and collateral declines in value, all losses can be adequately covered. Having sufficient collateral is particularly important to cover unexpected or extreme events that may threaten the viability of the organization.

During normal market conditions, we observe that some assets from the portfolio of collateral may exhibit losses while others may exhibit profits. The resulting diversification benefits allow the portfolio to have lower market risk than the sum of the market risk of the portfolio's components. Under normal conditions, the behaviour of the return distribution for securities can be characterized by one adverse effect. We call this the individual effect, since the individual securities may take values from low quantiles of the probability distribution of returns. While there are losses at the security level, at the portfolio level the dependence structure allows for diversification benefits. Therefore, market risk management practices during normal conditions generally focus on covering the risk associated with losses at the security level.

When extreme events occur — such as banking crises and sovereign debt defaultslarge values of collateral may need to be sold to cover financial losses. During these episodes, the behaviour of the return distribution for securities can be characterized by two adverse effects. In addition to the individual effect, the dependence structure may exhibit lower diversification benefits than those observed historically, and we call this the portfolio effect. ${ }^{1}$

In this paper, we propose strategies to manage the market risk that results from both the individual and portfolio effects. The implementation of the strategy to manage individual effects is largely based on Garcia and Gençay (2006). We recommend that both strategies be employed so that the market risk of the portfolio of collateral is managed during normal market conditions as well as during periods of extreme events.

To manage the market risk created by the individual effect, the strategy usually followed is to require collateral in excess of the exposure. The excess collateral is based on the characteristics of the asset pledged. These characteristics are captured

\footnotetext{
${ }^{1}$ Chan et al. (2005) refers to this as a phase locking effect. The authors offer an explanation for these effects from a financial engineering perspective.
} 
by a discount, often called a haircut. ${ }^{2}$ The strategy to mitigate risk associated with the individual effect requires a robust model for the tail of the return distribution, for each asset in the portfolio. For this model, we recommend the use of extreme value theory methods. While this strategy allows the risk manager to quantify the market risk associated with each individual asset in the portfolio of collateral and determine how much extra collateral is needed, it does not provide any information about the co-movement (or dependence) between the returns of the various assets that may be pledged. Knowing the dependence between the returns of these assets is important in order to assess the likelihood of bad outcomes (e.g., large negative returns) occurring at the same time. Capturing the dependence can be done by modeling the joint distribution of returns for the portfolio of assets pledged.

The portfolio effect may be linked to periods where there is a global flight to quality as a result of an extreme event, for example the Mexican peso crisis of 1994 and the Asian crisis of 1997. The dependence structures that existed before the crises are not indicative of the dependence structures in existence during the extreme event. To reduce the possible losses that may be associated with portfolio effects, we propose that portfolio managers actively select and hold those portfolios that have greater diversification at low quantiles of the return distribution. In addition, we recommend that for the existing portfolio, the degree of diversification at low quantiles is periodically monitored so that proper actions can be taken to maintain such a level of diversification. More specifically, we recommend that the following strategies be employed to manage the portfolio effects. First, when selecting among various possible assets that could be pledged as collateral, take those that have a lower probability of negative returns occurring simultaneously. Second, when monitoring the performance of an existing portfolio of collateral, calculate the probability associated with simultaneous losses occurring for all of the assets in the portfolio. Compare this calculation with pre-established thresholds for the probability of the event, and the associated losses. When the estimated probability and the associated losses breaches the pre-established thresholds, modify the composition of assets of the portfolio until the probability and associated losses are less than the pre-established threshold levels. ${ }^{3}$ Third, when stress testing the market value of a portfolio of collateral, include a scenario that imposes undesirable but plausible 'extreme' dependence structures, without changing the marginal return distributions.

\footnotetext{
${ }^{2}$ The haircut corresponds to a mapping from the loss distribution of returns into a single number; frequently this mapping is achieved using a Value-at-Risk measure. Garcia and Gençay (2006) explain the use of haircuts, and how to calculate them in the context of a securities settlement system. We do not elaborate on the different methodologies to calculate haircuts, instead we focus on the strategies that are designed to manage the portfolio effects.

${ }^{3}$ For example, for a portfolio of two assets, a risk manager may determine that adequate threshold levels for her institution are 5 per cent probability that each asset in the portfolio declines by 2 per cent. Should the estimated losses for each asset associated with a 5 per cent probability exceed the 2 per cent threshold, then the risk manager would recommend that the existing portfolio of collateral be replaced by another that has lower losses.
} 
The strategies to mitigate risk associated with the portfolio effect requires a framework to model the joint distribution of returns that is flexible enough to (i) capture the return characteristics of each asset; and (ii) capture the dependence structure. Specifically, the framework should allow the marginal distributions of the various assets to come from different families, and the dependence structure to depart from the assumption of multivariate normality. We propose a framework that achieves these two conditions, by relying on the the theory of copulas.

In this paper, we contribute to the literature on financial stability and risk management in two ways. First, we propose a strategy to manage market risks at the individual security level that accurately captures different tail behaviors of securities returns. This is accomplished using extreme value theory methods. Second, we propose strategies to manage market risk at the portfolio level that are able to capture a wide range of dependence structures departing from multivariate normal. The portfolio strategies take into account the properties of each asset and the dependence structure. These strategies are useful for (i) determining when to re-balance the collateral portfolio to assets that have a more favorable dependence structure during 'normal' periods; and for (ii) stress testing the market value of the collateral portfolio, to understand the potential impact of systemic events (e.g., stock market crashes) that are associated with a 'break-down' in the historical dependence structure. To present these strategies, we use data on securities pledged as collateral in the Canadian securities settlement system. Using these data we illustrate the implementation of these strategies.

There are benefits and costs of implementing these strategies. For example, the implementation costs of strategies designed to manage the individual effects are generally lower than those of portfolio effects since the former rely on modeling the univariate distribution of returns, a task that is well understood by risk managers. The costs of those strategies designed to manage portfolio effects are usually greater, since they require the dependence structure of the portfolio to be modeled, which is a more involved process. The main benefit of implementing risk management strategies that address both effects is that they provide a greater confidence level during normal and extreme events for the organization hedging risk. A secondary benefit is the resulting reduction in the volatility of portfolio returns.

The remainder of this paper is organized as follows. In section 2, we present copulas to study the dependence structure. In section 3, we use Canadian bond and equity data as a case study to present the proposed strategies and show how they may be implemented. In the final section we offer conclusions.

\section{Copula Approach}

Copulas are multivariate distributions that have proved to be extremely useful in financial engineering problems that involve modeling more than one random variable. The main advantage of copulas is that they separate the dependence structure 
from the marginal behaviour, and this separation is not possible with traditional representations of a multivariate density function. There are practical implications of this separability property that have made applications of copulas increasingly popular in a wide range of financial engineering problems. ${ }^{4}$ For example, one can fit a $t$-distribution to one variable and another distribution to the second, and then fit any copula across the marginals. In contrast, traditional multivariate distributions require that all the random variables have the same marginals. For example, if we fit a multivariate $t$ across a portfolio of two assets, we are forced to fit univariate $t$ s to each of the marginals. This restriction of traditional multivariate distributions becomes problematic when aggregating across assets that may have different distributions. Copulas not only provide a flexible way to model the marginals but also provide greater flexibility in that they permit a greater range of dependence structures to be imposed on the arbitrary marginals. Going back to our previous example, consider that we have a set of $t$ marginals, using the traditional multivariate representation only allows us one possible type of dependence structure, a multivariate $t$ distribution. Copulas instead allow us a greater range of additional dependence structures which include traditional multivariate representations. For these advantages we selected copulas as our framework to model the joint distribution for a portfolio of collateral. In the next section, we provide a concise review of copulas.

\section{$2.1 \quad$ Review}

Two components are necessary to model the joint return distribution of a portfolio of assets. The first is a model for the marginal distribution of each asset, and the second is a model for the dependence structure between the assets. There are several ways to model the marginal distribution. These include parametric methods that estimate the necessary parameters to characterize a given distribution, and non-parametric approaches that directly use the empirical distribution. Along with choosing one of the above mentioned approaches, the estimation of the joint distribution requires an approach to model for the dependence structure. As we have mentioned above, copulas are a flexible approach to model a wide range of dependence structures.

Copulas are multivariate distribution functions with standard uniform marginal distributions. A $d$-dimensional copula is represented as follows,

$$
C(u)=C\left(u_{1}, \cdots, u_{d}\right)
$$

where $u_{1}, \ldots, u_{d}$ are standard uniform marginal distributions. ${ }^{5}$ Copulas are a par-

\footnotetext{
${ }^{4}$ Copulas have been applied in credit risk modeling, portfolio management, derivatives pricing, risk aggregation, and the estimation of risk measures.

${ }^{5}$ There are three necessary conditions for a copula $C(u)$ to be a probability distribution: (i) the cumulative distribution functions must always be increasing in each component $u_{i}$, (ii) the marginal component $i$ can be obtained by setting $u_{j}=1$ for all $j \neq i$ and must be uniformly distributed, and (iii) the joint probability is non-negative. For the mathematical expressions of these conditions, we refer the reader to Zivot and Wang (2006).
} 
simonious approach to model multivariate distributions with arbitrary marginals. This is possible due to the Sklar (1959) theorem which states that copulas may be constructed in conjunction with univariate distribution functions to build multivariate distribution functions. In working with copulas, one can build multivariate distributions using probability transformations to map arbitrary marginals into standard uniform marginals and then choose a copula function that captures the dependence structure between the marginals. Also one can backtrack the initial (arbitrary) marginals from the copula by applying quantile transformations to the copula's uniform marginals.

In Figure 1, we illustrate the flexibility of copulas to capture the dependence of the data. The left panel of Figure 1 presents the scatter plot of actual returns for two assets $r_{1}$ and $r_{2}$ that correspond to Royal Bank of Canada and Bank of Nova Scotia common stock. The right panel depicts the returns obtained from fitting a copula to the actual return data.
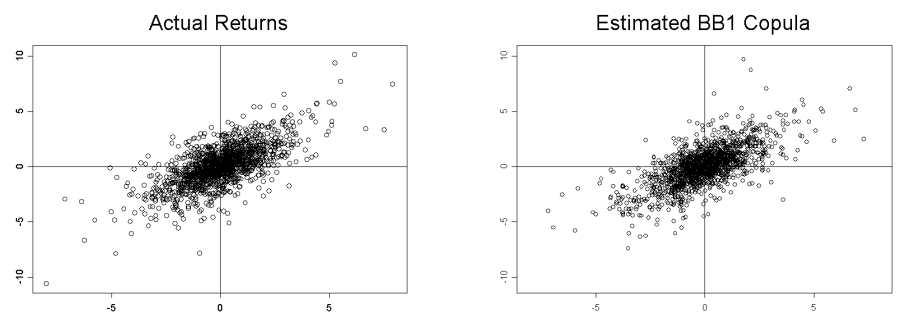

Figure 1: Scatter Plots: Actual Data And Fitted Copula

These figures show scatter plots of daily returns for Royal Bank of Canada $\left(r_{1}\right)$, and Bank of Nova Scotia $\left(r_{2}\right)$. Actual returns are depicted in the left panel, and those fitted using a copula are depicted in the right panel.

\subsection{Types of copulas}

To model the dependence structure between the marginal return distributions, we need to select among various types of possible copulas. These copulas involve different types of dependence structures. ${ }^{6}$ We classify copulas into three groups: fundamental, implicit and explicit copulas. ${ }^{7}$ For all the copulas presented in this section, $u_{1}$ and $u_{2}$ represent standard uniform marginals.

\subsubsection{Fundamental copulas}

Fundamental copulas can be used to model marginals that exhibit one of three structures: perfect negative dependence, independence, or perfect positive dependence.

\footnotetext{
${ }^{6}$ For a more exhaustive listing of copulas and their characteristics see Zivot and Wang (2006).

${ }^{7}$ We use bivariate copulas to present the different types.
} 
These copulas result from the statistical concept of Fréchet bounds, which states that the joint probability is constrained within precise bounds,

$$
\max \left(F_{x_{1}}+F_{x_{2}}-1,0\right) \leq F\left(x_{1}, x_{2}\right) \leq \min \left(F_{x_{1}}, F_{x_{2}}\right)
$$

where $\max \left(F_{x_{1}}+F_{x_{2}}-1,0\right)$ corresponds to the lower Fréchet bound and $\min \left(F_{x_{1}}, F_{x_{2}}\right)$ corresponds to the upper Fréchet bound. Based on this result, it is natural to use these bounds as cases of extreme dependency and define the minimum copula (perfect negative dependence case) by,

$$
C\left(u_{1}, u_{2}\right)=\max \left(u_{1}+u_{2}-1,0\right)
$$

and maximum copula (perfect positive dependence case) by,

$$
C\left(u_{1}, u_{2}\right)=\min \left(u_{1}, u_{2}\right)
$$

The third fundamental copula captures the case of independent random variables. This is the product copula and it is defined as follows:

$$
C\left(u_{1}, u_{2}\right)=u_{1} \cdot u_{2}
$$

\subsubsection{Implicit copulas}

Existing multivariate distributions such as the multivariate normal and multivariate $t$ describe important dependence structures. The same dependence structures specified by these distributions can be captured in a more general way by copulas. For example, the multivariate normal gives rise to the normal copula, and the multivariate $t$ to the $t$-copula. For example, a bivariate normal copula can be expressed as,

$$
C_{\Sigma}^{N}=\Phi_{\Sigma}\left(\Phi^{-1}\left(u_{1}\right), \Phi^{-1}\left(u_{2}\right)\right)
$$

where $\Phi$ denotes the probability distribution of a standard normal random variable while $\Phi_{\Sigma}$ denotes the probability distribution of a normal random variable with mean zero and covariance matrix $\Sigma$. A bivariate normal copula can represent dependence structures that interpolate from the maximum and minimum copula (including the product copula), where the linear correlation coefficient represents the strength of the dependence. Similarly a $t$-copula can be expressed as,

$$
C_{v, \Sigma}^{t}=t_{v, \Sigma}\left(t_{v}^{-1}\left(u_{1}\right), t_{v}^{-1}\left(u_{2}\right)\right)
$$

where $t_{v}$ denotes a $t$ probability distribution with $v$ degrees of freedom. The $t$ copula can be used instead of a normal copula in situations where it is known that the marginal distributions have fatter tails than the normal. As it is shown for the case of the normal- and $t$-copula, implicit copulas can be extracted from well-known distributions. 


\subsubsection{Explicit copulas}

Explicit copulas are obtained using simple closed form expressions. ${ }^{8}$ One group of explicit copulas are Archimedean copulas which are all defined using the following expression,

$$
C\left(u_{1}, u_{2}\right)=\phi^{-1}\left(\phi\left(u_{1}\right)+\phi\left(u_{1}\right)\right)
$$

where $\phi$ is called the generator function which is different for each type of Archimedean copula. The generator function is a decreasing function with a domain on $[0,1]$ and a rank on $[0, \infty]$. There are a number of different Archimedean copulas that exist; we report four commonly used ones in Table 1.

\begin{tabular}{lll}
\hline Name & Copula & Parameter Range \\
\hline Clayton & $\left.C\left(u_{1}, u_{2}\right)=\left(u_{1}^{-\theta}+u_{2}^{-\theta}-1\right)^{-\frac{1}{\theta}}\right)$ & $0<\theta<\infty$ \\
Gumbel & $C\left(u_{1}, u_{2}\right)=e^{-\left[\left(-\ln u_{1}\right)^{\theta}+\left(\ln u_{2}\right)^{\theta}\right]^{\frac{1}{\theta}}}$ & $1 \leq \theta<\infty$ \\
Frank & $C\left(u_{1}, u_{2}\right)=\frac{1}{\theta} \ln \left(1+\frac{\left(e^{\theta u_{1}}-1\right)\left(e^{\theta u_{2}}-1\right)}{e^{\theta}-1}\right)$ & $-\infty<\theta<\infty$ \\
BB1 & $C\left(u_{1}, u_{2}\right)=\left(1+\left[\left(u_{1}^{-\theta}-1\right)^{\delta}+\left(u_{2}^{-\theta}-1\right)^{\delta}\right]^{-1 / \theta}\right)$ & $\theta>0, \delta \geq 1$ \\
BB4 & $C\left(u_{1}, u_{2}\right)=\left(u_{1}^{-\theta}+u_{2}^{-\theta}-1-\left[\left(u_{1}^{-\theta}-1\right)^{-\delta}+\left(u_{2}^{-\delta}-1\right)^{-\delta}\right]^{-1 / \delta}\right)^{-1 / \theta}$ & $\theta \geq 0, \delta>0$ \\
\hline
\end{tabular}

Table 1: Archimedean Copulas

This table presents various commonly used Archimedean copulas. These copulas capture various types of dependence ranging from lower tail dependence (Clayton), to perfect positive dependence (Gumbel).

The Clayton (1978) copula, also known as the Kimeldorf and Sampson (1975) copula, is useful to represent dependence structures that exhibit only lower tail dependence. The Gumbel copula can be used to represent dependence structures that interpolate from the case of no dependence $(\theta=1)$ to the case of perfect dependence $(\theta \rightarrow \infty)$. The Gumbel copula is also said to belong to the class of extreme value (EV) copulas. EV copulas are those where the following scaling property holds,

$$
C\left(u_{1}^{\delta}, u_{2}^{\delta}\right)=\left(C\left(u_{1}, u_{2}\right)\right)^{\delta}
$$

where $\delta>0$ and Equation 9 holds for all $\left(u_{1}, u_{2}\right) \epsilon[0,1] \cdot[0,1]$. Durrleman et al. (2000) is a good reference for EV-copulas and their relationship with extreme value theory. The Frank copula does not exhibit lower or upper tail dependence, whereas the BB1 copula and BB4 are able to capture tail dependence. The BB4 copula also belongs to the class of Archimax copulas which is a combination of extreme value and Archimedean copulas.

\footnotetext{
${ }^{8}$ We focus on the Archimedean copulas and do not cover the Marshall-Olkin copulas. For the interested reader we recommend Embrechts et al. (2001) and Schmidt (2006) as excellent references.
} 


\subsection{Choosing the appropriate copula}

There are various visual techniques available for studying the dependence structure between the return distributions of two assets. These techniques are used to identify the type of dependence structure that exists in the historical return time series. Drawing from the information provided by these techniques, we can determine the appropriate copula model for the data. Two of these techniques are scatter plots of returns, and level plots. A scatter plot of returns is useful to visualize the dependence structure at different quantiles. Of particular importance for risk management, is the determination of whether or not there is any evidence of lower tail dependence (i.e. simultaneous losses). The fundamental copulas can be used as benchmark cases where one can compare their scatter and level plots with those of the data. This comparison can help to identify the type of dependence in the data. For example, the minimum copula exhibits negative dependence, the independence copula no dependence, and the maximum copula positive dependence. One may note that the maximum copula represents the riskiest case because it involves simultaneous losses in both assets. Scatter plots for the minimum, independent, and maximum copulas are shown in the first row of Figure 2 in the left, middle, and right panels respectively.

A level plot is another technique that is used to visualize the dependence structure between returns. Level plots for the three fundamental copulas (minimum, independent, and maximum) are shown in the second row of Figure 2 in the left, middle, and right panels. In Figure 2 the minimum copula is characterized by a linear negative relationship, the maximum copula is characterized by sharp angles, and the independent copula is characterized by a positive convexity. Using scatter

and level plots on the historical data are useful in suggesting what type of copulas may provide an accurate fit to the data. 

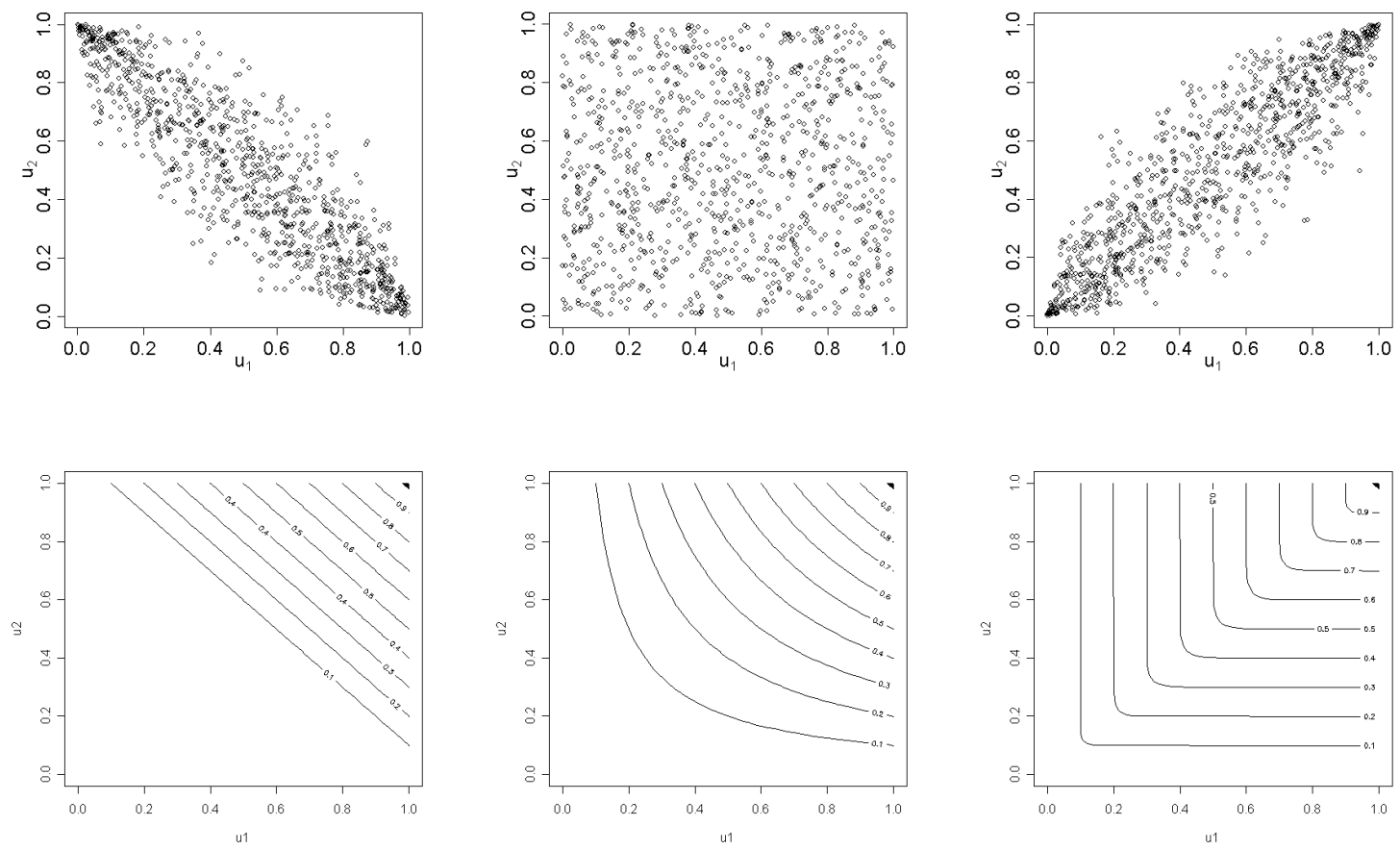

Figure 2: SCATTER AND LEVEL PLOTS

From left to right, the panels in the first row show the scatter plot of the marginals for the minimum, independent and maximum bivariate fundamental copulas. The second row shows the level plots for the minimum, independent and maximum bivariate fundamental copulas.

In section 3.3.2, we present the copula approach to estimate the joint return distribution for a portfolio of two assets that could be pledged as collateral in Canada's securities clearing and settlement system. Subsequently, we construct various portfolios of equities and bonds, estimate the joint return distribution, and implement the proposed risk management strategies.

\section{Risk Management Strategies for Bond and Eq- uity Portfolios}

To manage the market risk for a portfolio of collateral we propose strategies designed to manage the market risk associated with losses of each asset (previously defined as individual effect), and strategies to address the market risk associated with changes in the dependence structure between the assets of the portfolio (previously defined as portfolio effect). We use data from equities and bonds that are pledged in Canada's securities clearing and settlement system to show how these strategies can be implemented. These strategies are as follows: 


\section{- Strategy for individual effects}

1. For each asset in the portfolio, discount (or haircut) its market value. This haircut should reflect the market risk of the asset and should be used to require a greater amount of collateral so that a given exposure is covered with greater certainty.

This strategy requires the estimation of haircuts, which consists of selecting a model of the distribution of losses as well as a risk measure. ${ }^{9}$

\section{- Strategies for portfolio effects}

1. To select among various portfolios of collateral, calculate the probability of observing simultaneous losses. Select the portfolio that has the lowest joint probability of exhibiting joint losses.

2. When monitoring an existing portfolio of collateral, set a threshold level for: (i) the probability that losses for all assets in the portfolio occur at the same time, and (ii) the magnitude of these losses. Regularly calculate this probability for the portfolio and check that the associated losses are less than the established threshold.

3. Stress test the dependence structure of the portfolio. Capture the effect of the different scenarios by estimating the resulting haircut for the portfolio. Construct these scenarios by imposing copulas that correspond to adverse dependence structures, and for each copula calculate the haircut for various degrees positive dependence.

These strategies require the estimation of the joint distribution of returns. We do this using a copula approach. This approach can be thought of as a two-step process. The first consists of finding a model for the marginal return distributions, and the second consists of finding a copula that fits the dependence structure of the data. An example of the implementation of this two-step process is presented in section 3.2 for a portfolio of two equities, and by, following this, the process is replicated for other (equities and fixed income) portfolios created from randomly sampling our data set.

\subsection{Data}

Our sample data consists of securities that are eligible to be pledged in the Canadian securities clearing and settlement system. These data include bonds and equities. The bond data are obtained from the Bank of Canada, and contains the closing secondary market bond prices (bid, offer, mid) and yields (mid) for all Canadian

\footnotetext{
${ }^{9}$ Garcia and Gençay (2006) present various combinations of parametric and non-parametric methods to estimate the loss distribution and various risk measures to estimate haircuts.
} 
dollar denominated Government of Canada domestic marketable bonds auctioned by the Bank of Canada on behalf of the Minister of Finance. This data contains 250 bond securities. For our analysis, we focus on bid prices as a proxy for the price that could be realized should these securities need to be sold. Using the bid price we calculate the returns for each security as the daily percentage changes. We classify the bid returns in five groups based on their maturity; the corresponding descriptive statistics are found in Table 2.

\begin{tabular}{lccccr}
\hline Maturity & \# of Series & \multicolumn{2}{c}{ Skewness $<0$} & \multicolumn{2}{c}{ Kurtosis $>3$} \\
& & \# of Series & $\%$ & \# of Series & $\%$ \\
\hline$<1$ & 16 & 5 & $31.3 \%$ & 12 & $75 \%$ \\
1 to 3 & 71 & 46 & $64.8 \%$ & 71 & $100 \%$ \\
3 to 5 & 31 & 8 & $25.8 \%$ & 31 & $100 \%$ \\
5 to 10 & 59 & 30 & $50.8 \%$ & 58 & $98.3 \%$ \\
$>10$ & 73 & 27 & $37.0 \%$ & 71 & $97.3 \%$ \\
\hline
\end{tabular}

Table 2: Bond Bid Returns: Descriptive Statistics

This table shows the descriptive characteristics for secondary market bid returns for selected Government of Canada domestic marketable bonds. The data includes bonds with a maturity starting in 1988 and up. Source: dmb database, Bank of Canada, Financial Market Department.

In Table 2 we observe that a large percentage of securities exhibit negative skewness, and excess kurtosis with respect to a normal distribution. In Table 3 we present the statistical properties for each maturity class by constructing a single vector of returns per class.

\begin{tabular}{lcccc}
\hline Maturity & Mean & Std. Dev. & Skewness & Kurtosis \\
\hline 0 to 1 & 0.0024 & 0.0784 & 0.3506 & 41.36 \\
1 to 3 & -0.0004 & 0.1041 & -0.4063 & 21.56 \\
3 to 5 & -0.0014 & 0.1933 & 0.0536 & 13.50 \\
5 to 10 & 0.0020 & 0.2666 & 0.4034 & 64.28 \\
$>10$ & 0.0042 & 0.3902 & 0.0830 & 17.27 \\
\hline
\end{tabular}

\section{Table 3: Bond Bid Returns: Group Descriptive Statistics}

This table shows the statistical characteristics for secondary market bid returns for selected Government of Canada domestic marketable bonds. The data includes bonds with a maturity starting in 1988 and up. Source: dmb database, Bank of Canada, Financial Markets Department.

Table 3 shows that for all groups there is evidence of positive skewness, with the exception of the group that contains securities with a maturity from 1 to 3 years. As for kurtosis, all groups exhibit excess kurtosis.

The equity data is obtained from the Canadian Depository for Securities (CDS) and consists of closing stock prices for 130 equity instruments that constitute a representative sample of securities that are acceptable as collateral in the system. CDS imposes sector limits per asset class for eligible collateral; this is done to encourage diversification in the portfolio of collateral. For the case of equities, each participant is allowed a maximum of $\$ 100$ million which suggests - given the 
large values that must be collateralized - that the share of equities from the overall collateral portfolio is small. The sample period for the equities data is from 5 January 1998 to 12 March 2004. For all equity returns there is excess kurtosis and 58 of the 131 equities exhibit negative skewness.

The statistical properties for both bond and equity returns suggest that fat tails in the return distribution are common and therefore, appropriate methods - such as those based on extreme value theory - should be used to estimate the return distributions.

\subsection{Methodology}

To present the methodology, we use equity return data for two assets in our database: Royal Bank of Canada common stock (asset 1), and Bank of Nova Scotia common stock (asset 2) which from now on we jointly refer to as portfolio 1. Both of these securities are among the acceptable assets that can be pledged as collateral in Canada's securities clearing and settlement system.

We first present the methodology to implement the risk management strategy that addresses individual effects. This requires us to model the marginal distributions of returns. Next we present the methodology to implement the strategies that address portfolio effects. The latter strategies require a model for the marginal distributions, as well as a model for the dependence structure.

\subsubsection{Strategy for individual effects: Estimating marginal return dis- tributions}

To model the marginal return distributions we first create a $q q$-plot (quantilequantile plot) to examine whether the sample returns for both assets come from a specific distribution. Specifically, we compare the empirical quantiles with those of a normal distribution. We use the normal as the benchmark case for a thin tailed distribution. If the returns can be in fact modeled with the hypothesized distribution (i.e. normal), the $q q$-plot should be linear. 

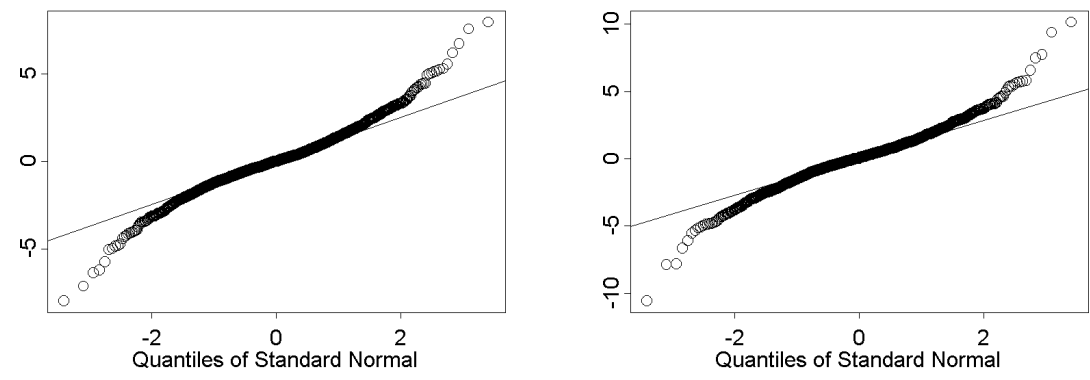

Figure 3: QQ-PLOTS OF RETURNS

The left panel shows the $q q$-plot of the return distribution for asset 1 (portfolio 1). The right panel shows the corresponding $q q$-plot for asset 2 (portfolio 1). The $q q$-plots compare the quantiles of the sample return distributions with those of a normal distribution.

Figure 3 shows that for both returns, the $q q$-plot indicates that the empirical distribution comes from a fat-tailed distribution. We confirm these results by applying the Jarque-Bera test on the sample returns. This is shown in Table $4 .{ }^{10}$

\begin{tabular}{|c|c|c|}
\hline & \multicolumn{2}{|c|}{ EQUITY PORTFOLIO 1} \\
\hline & Asset 1 & Asset 2 \\
\hline Test statistic & 357.46 & 478.26 \\
\hline$p$-value & 0.00 & 0.00 \\
\hline
\end{tabular}

Table 4: TEST OF NORMALITY

This table presents the results of Jarque-Bera tests on the return sample distributions for asset 1 and asset 2 in portfolio 1 . The distribution under null is a chi-square with 2 degrees of freedom. The total number of observations is 1482 .

Table 4 shows that we can reject the null hypothesis that the individual returns are normally distributed. Since the marginal distributions of the two return series are not normal, we model the tails of the marginal return distributions using a generalized Pareto distribution (GPD), and for the center of the distribution, we use non-parametric techniques based on the empirical distribution.

\footnotetext{
${ }^{10}$ We corroborated the results of the Jarque-Bera (JB) test with the Shapiro-Wilks (SW) test. The SW test statistic for asset 1 is 0.9758 (p-value 0.0000) and for asset 2 is 0.9794 (p-value 0.0000).
} 

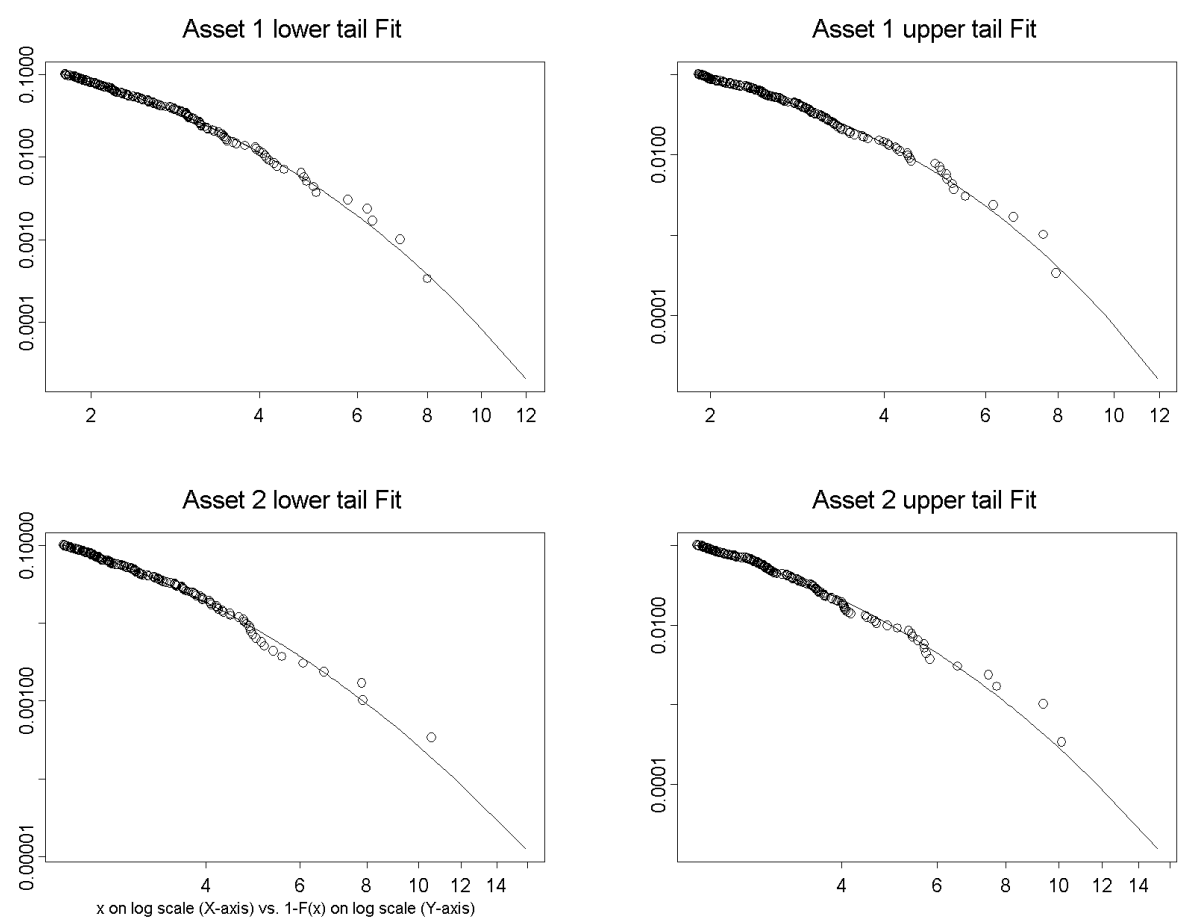

Figure 4: Fit OF THE TAILS

This figure shows on the first row the estimated models for the lower and upper tail of asset 1 (represented by a smooth line) and the actual observations (represented by points). The second row shows the estimated models for the lower and upper tail for asset 2 and the actual observations.
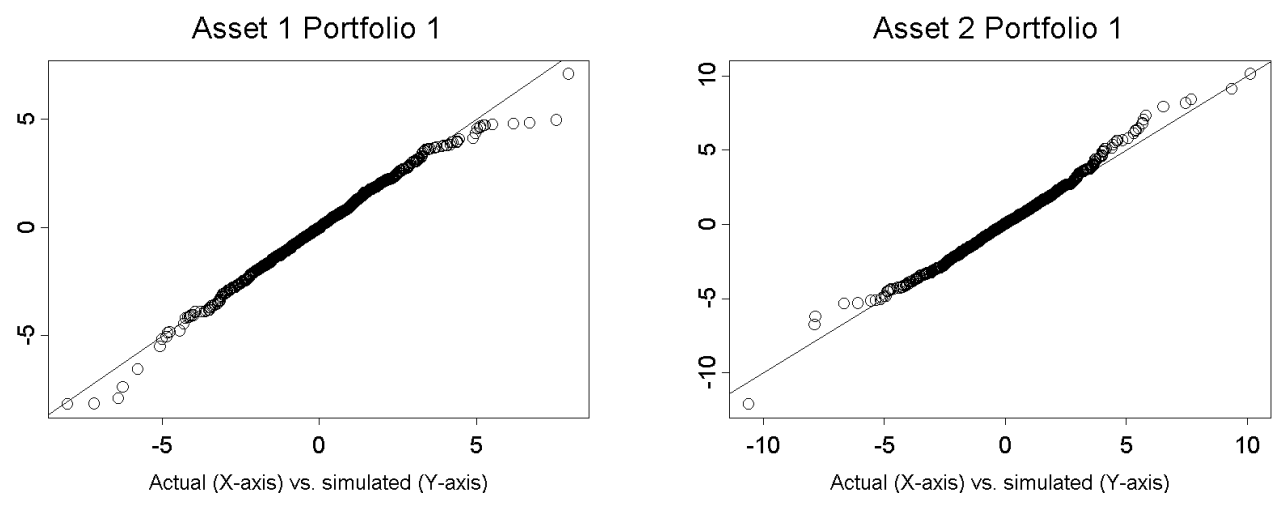

Figure 5: Fit of the SemiparametriC GPD MARginals

This figure shows two panels for the $q q$-plots of the semi-parametric GPD model and the data. The $q q$-plots show a linear relationship which provides evidence that the semi-parametric GPD marginal provides a good fit to the marginal distributions of portfolio 1. 
This semi-parametric approach is based on Carmona (2004), and Zivot and Wang (2006). Note that other approaches may be followed to capture the fat tails. For example one could use $t$-distributions to model the marginals or one can make use of extreme value theory techniques. We follow the latter approach. For the estimation of the tails we choose lower and upper threshold values of -1.78 and 1.88 per cent for asset 1, and lower and upper threshold values of -2.16 and 2.16 for asset 2 . Using these thresholds the semi-parametric GPD models are estimated. To evaluate the fit of the tails, we create plots of the excesses over the specified lower and upper thresholds against the quantiles of the fitted GPD model, as shown in Figure 4. Figure 4 indicates that there is a good fit of the tails of the marginal return distributions. ${ }^{11}$

Similarly we can observe the overall fit of the semi-parametric GPD marginals in Figure 5. The linearity of the $q q$-plots shown in Figure 5 confirms that the semiparametric GPD models adequately describe the marginal return distributions.

\subsubsection{Strategy for portfolio effects: Estimating the dependence struc- ture}

We now model the dependence structure for the semi-parametric generalized Pareto distribution (GPD) marginals. We start by constructing a scatter plot of the historical returns for the assets of portfolio 1. This scatter plot is shown in the top left panel of Figure 6 where we observe the presence of a moderately positive dependence in the return data. We also notice that there are joint realizations at the lower and upper quantiles of the return distributions that require selecting a copula that can capture these observations. Note that if we create a scatter plot of a bivariate normal model calibrated to the data depicted in the top right panel of Figure 6, we observe that the bivariate normal model is not able to replicate well the lower and upper dependence observed in the return data. Also note that the two copulas (normal and BB1 in the lower panels) with semiparametric marginals provide a closer fit to the actual returns.

\footnotetext{
${ }^{11}$ Standard tools in extreme value theory such as mean excess plots are used to select adequate values for the corresponding thresholds.
} 

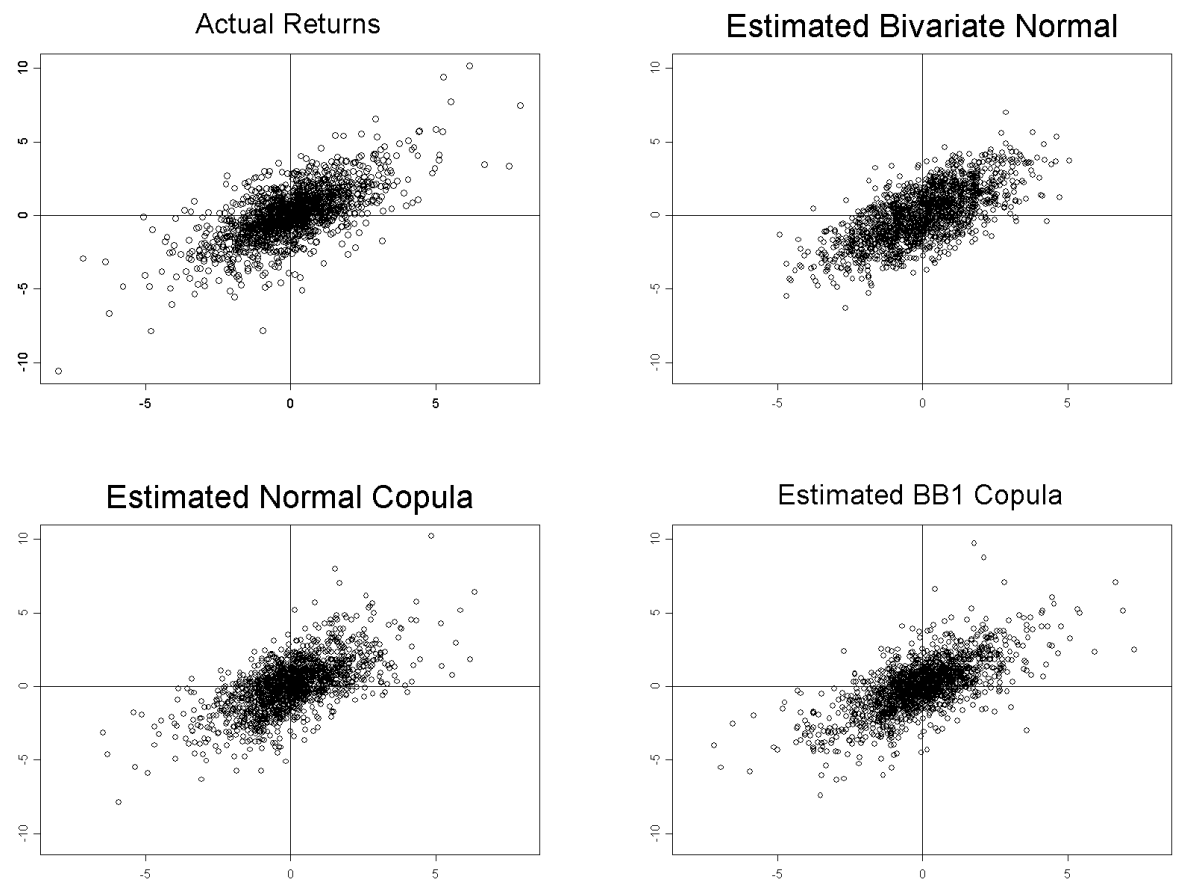

Figure 6: SCATTER PlOT FOR THE DATA, A BIVARIATE NORMAL MODEL, A NORMAL COPULA, AND A BB1 COPULA

These figures show scatter plots for the returns of portfolio 1. TOP LEFT PANEL: Shows the actual returns. TOP RIGHT PANEL: Shows returns coming from a bivariate normal with the correlation of the data and the mean and standard deviation of the corresponding returns. BotTOM LEFT PANEL: Shows returns coming from a normal copula with semi-parametric GPD marginals. Bоттом RIGHT PANEL: Shows returns coming from a BB1 copula with semi-parametric GPD marginals.

We now proceed to estimate the copula models following the approach proposed by Zivot and Wang (2006). This requires us to calculate an empirical copula and use maximum likelihood to obtain the parameters associated with the copula model. We use five potential copula models to estimate the dependence structure of the returns, which are: the normal copula, the Gumbel copula, the Frank copula, the BB1 copula, and the BB4 copula. Our choice of copulas is not exhaustive. We select five copulas that are commonly used and are able to capture various types of dependence. To select the appropriate copula among the various possibilities, we compare the log-likelihood value of the fitted copula model. These results are shown in Table 5. 


\begin{tabular}{lc}
\hline & Log-likelihood \\
\hline Normal copula & 443.2 \\
Gumbel copula & 439.1 \\
Frank copula & 400.6 \\
BB1 & 466.4 \\
BB4 & 463.9 \\
\hline
\end{tabular}

Table 5: ChoOsing THE COPUlA

This table presents the log-likelihood fit of five competing copula models. The results indicate that the BB1 copula is the best fit.
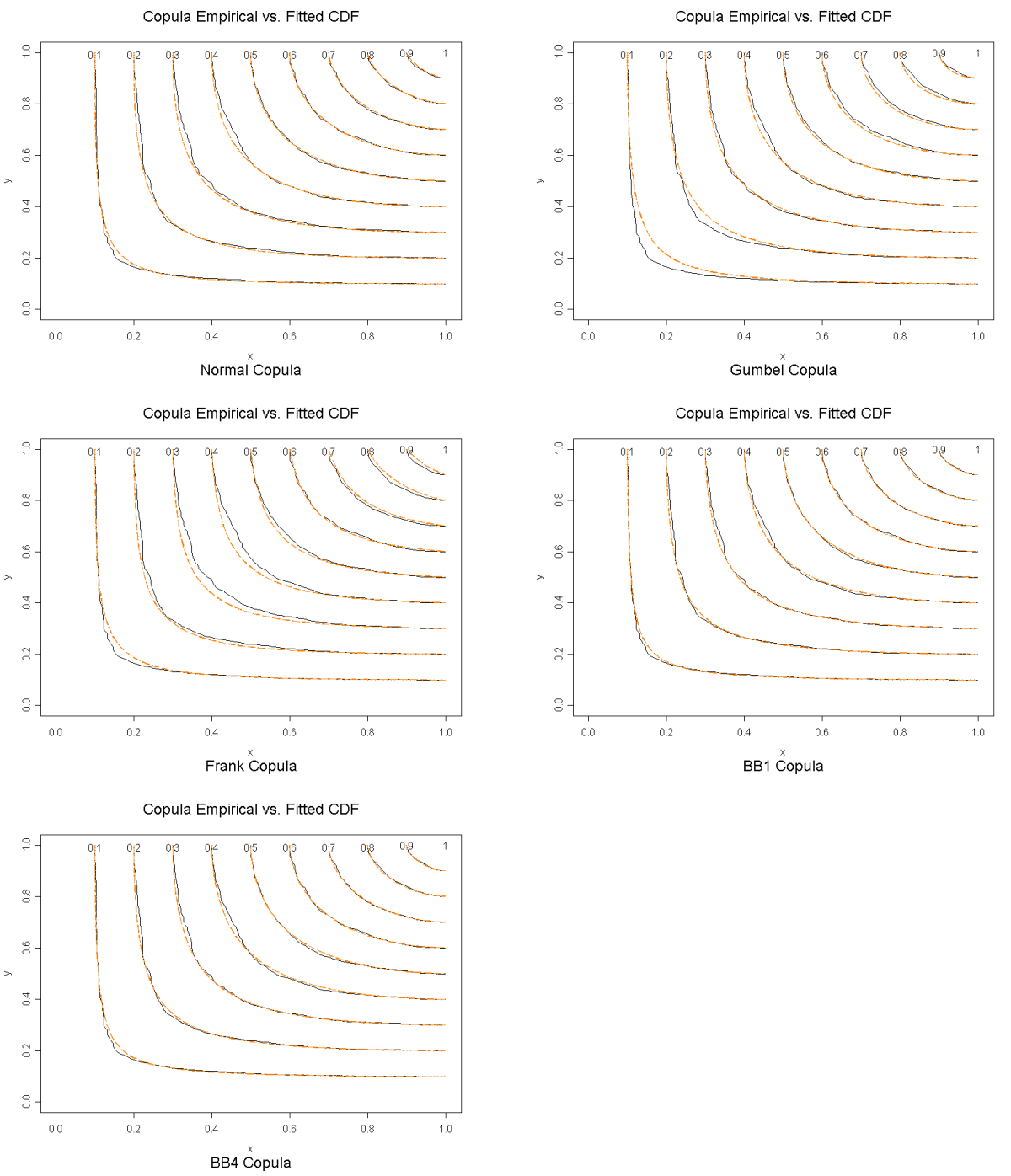

Figure 7: Level Plots: Copula Model Fit

The five panels show both level plots for the return data for asset 1 and asset 2 (black line) and level plots for the copula that is fitted to the data (orange dotted line). Observing the panels, we corroborate that BB1 copula is a closer fit to the dependence structure of data. 
We also calculate level plots for the empirical copula and the fitted copula models to visually determine which model is a better fit. This is shown in Figure 7. Both results presented in Table 5 and Figure 7 suggest that the BB1 copula provides a better fit to the dependence in the data. Also by constructing the scatter plot of the estimated BB1 copula (shown in the bottom right panel of Figure 6) we observe that the BB1 copula with semi-parametric GPD marginals is able to reproduce the lower and upper dependence behaviour observed in the data.

\subsection{Implementing risk management strategies}

We now apply the methodology outlined in section 3.2 to various portfolios and report the results of implementing these strategies. ${ }^{12}$

\subsubsection{Strategy for individual effects}

\section{Strategy 1: Apply haircuts to determine collateral requirements}

To implement this strategy, we require a model of the lower tail (losses) of the marginal return distributions. We model the tail with a generalized Pareto distribution (GPD) and estimate the haircuts. The GPD methodology provides a good approximation of various different tail behaviors (e.g., thin, fat or finite tail). ${ }^{13}$

Using the GPD methodology, the haircut for asset 1 using a 99 per cent confidence level corresponds to 4.09 per cent with a Value-at-Risk measure, and 5.23 per cent with an expected shortfall measure. For asset 2 the haircuts also using a 99 confidence interval correspond to 4.68 per cent with a Value-at-Risk measure, and 6.01 per cent with an expected shortfall measure. ${ }^{14}$

An interesting comparison to make is to calculate the haircut that would result for the portfolio. We do this assuming an equally weighted portfolio. For such a portfolio, the associated haircuts are 4.01 per cent when Value-at-Risk is used, and 5.06 per cent when expected shortfall is used. As it is expected, these results confirm that when haircuts are calculated on a asset-by-asset basis they exceed the haircut that would be required for the entire portfolio. This result makes the total

\footnotetext{
${ }^{12}$ The portfolios studied are constructed by randomly drawing securities without replacement from the dataset.

${ }^{13}$ A theorem by Balkema and de Haan (1974) and Pickands (1975) shows that for a sufficiently high threshold, the distribution function of the excess may be approximated by the GPD, because as the threshold gets large, the excess distribution converges to the GPD.

${ }^{14}$ The expected shortfall (ES) of an asset or a portfolio is the average loss given that Valueat-Risk has been exceeded. For example, the $\alpha$ per cent ES is the conditional mean of negative returns $r_{t}$, given that $r_{t}>\operatorname{Va} R_{t}(\alpha)$ :
}

$$
E S_{t}(\alpha)=E\left[r_{t} \mid r_{t}>\operatorname{VaR}(\alpha)\right]
$$


collateral resulting from individual haircuts cover a greater confidence level than the confidence level calculated for the entire portfolio. This difference is due to the diversification effects at the portfolio level. It is this difference that may be eliminated during extreme events due to large liquidation of portfolios which lead the correlations to become much greater. To reduce the probability of entirely losing the diversification benefits we propose the following strategies.

\subsubsection{Strategies for portfolio effects}

\section{Strategy 1: Select those portfolios that exhibit a lower probability of simultaneous losses}

To implement this strategy we require a model for the marginals, and a model for the joint distribution. These models are obtained using the extreme value theory methods for the marginals and a copula approach for the joint distribution. We apply this strategy to portfolios of two securities consisting of equities or bonds where each asset accounts for 50 per cent of the portfolio. For all the portfolios we compare the fit of a normal copula, a Gumbel copula, a Frank copula, and BB1 copula, and a BB4 copula. The estimation of the copula is carried out by maximizing the likelihood function.

Having a model for the marginals and dependence allows us to estimate the probability of simultaneous losses for both assets in each portfolio. Table 6 shows the probabilities of observing simultaneous equal losses for both assets of each of the randomly selected portfolios of equities. The corresponding results for bond portfolios are shown in Table 7. A more detailed analysis of Tables 6 and 7 allows us to identify the portfolios with the lowest joint probability of simultaneous losses. For example, among the equity portfolios, portfolio 28 has the lowest probability $(1.8$ per cent) of observing a 1 per cent simultaneous loss in both assets, and portfolio 25 has the lowest probability (0.19 per cent) of observing a 2 per cent simultaneous loss in both assets.

One result that stands out, is that bond portfolios exhibit much lower probabilities of joint losses than equities. Comparing Tables 6 and 7, we observe that bond portfolios exhibit probabilities of simultaneous losses of practically zero for losses of 2 per cent or more, whereas equity portfolios exhibit probabilities with greater values. This result suggests that operators of financial infrastructures that take a large proportion of these particular bond portfolios are better protected against simultaneous losses, when compared to other operators who may take mostly equities as collateral. 


\begin{tabular}{|c|c|c|c|c|c|c|c|}
\hline \multicolumn{8}{|c|}{ PORTFOLIOS OF EQUITIES } \\
\hline & \multicolumn{7}{|c|}{ Simultaneous Losses } \\
\hline & Assets & Copula & 1 per cent & 2 per cent & 3 per cent & 4 per cent & 5 per cent \\
\hline Portfolio $_{1}$ & 2,3 & BB1 & 0.1272 & 0.0448 & 0.0150 & 0.0055 & 0.0022 \\
\hline Portfolio $_{2}$ & 126,103 & Normal & 0.0247 & 0.0025 & 0.0003 & 0.0001 & 0.0000 \\
\hline Portfolio $_{3}$ & 65,46 & BB1 & 0.0796 & 0.0218 & 0.0069 & 0.0024 & 0.0009 \\
\hline Portfolio $_{4}$ & 72,62 & BB1 & 0.0183 & 0.0020 & 0.0002 & 0.0000 & 0.0000 \\
\hline Portfolio5 & 14,122 & BB1 & 0.0874 & 0.0258 & 0.0086 & 0.0029 & 0.0010 \\
\hline Portfolio $_{6}$ & 45,61 & BB1 & 0.0877 & 0.0227 & 0.0062 & 0.0018 & 0.0006 \\
\hline Portfolio7 & 37,49 & BB4 & 0.0593 & 0.0120 & 0.0035 & 0.0013 & 0.0006 \\
\hline Portfolio $_{8}$ & 2,124 & BB1 & 0.0733 & 0.0169 & 0.0043 & 0.0013 & 0.0004 \\
\hline Portfoliog & 103,130 & Frank & 0.1704 & 0.0703 & 0.0312 & 0.0125 & 0.0052 \\
\hline Portfolio 10 & 122,8 & BB1 & 0.0828 & 0.0269 & 0.0092 & 0.0034 & 0.0014 \\
\hline Portfolio11 & 60,127 & Frank & 0.0707 & 0.0136 & 0.0016 & 0.0000 & 0.0000 \\
\hline Portfolio12 & 32,16 & BB1 & 0.0918 & 0.0217 & 0.0069 & 0.0028 & 0.0013 \\
\hline Portfolio $_{13}$ & 30,86 & BB1 & 0.0937 & 0.0315 & 0.0126 & 0.0048 & 0.0021 \\
\hline Portfolio14 & 8,2 & BB1 & 0.0745 & 0.0172 & 0.0045 & 0.0013 & 0.0004 \\
\hline Portfolio15 & 77,15 & BB1 & 0.0912 & 0.0223 & 0.0052 & 0.0015 & 0.0005 \\
\hline Portfolio16 & 15,61 & BB1 & 0.1083 & 0.0310 & 0.0084 & 0.0027 & 0.0010 \\
\hline Portfolio $_{17}$ & 51,2 & BB1 & 0.0957 & 0.0263 & 0.0079 & 0.0027 & 0.0010 \\
\hline Portfolio18 & 84,127 & BB4 & 0.0719 & 0.0127 & 0.0021 & 0.0003 & 0.0000 \\
\hline Portfolio19 & 56,50 & BB1 & 0.1390 & 0.0546 & 0.0233 & 0.0109 & 0.0055 \\
\hline Portfolio $_{20}$ & 68,114 & $\mathrm{BB} 1$ & 0.1754 & 0.0905 & 0.0398 & 0.0194 & 0.0095 \\
\hline Portfolio25 & 14,81 & Frank & 0.0240 & 0.0019 & 0.0002 & 0.0000 & 0.0000 \\
\hline Portfolio26 & 104,70 & BB1 & 0.0432 & 0.0075 & 0.0015 & 0.0003 & 0.0001 \\
\hline Portfolio $_{27}$ & 120,125 & Frank & 0.1272 & 0.0419 & 0.0159 & 0.0058 & 0.0022 \\
\hline Portfolio 28 & 42,81 & BB4 & 0.0180 & 0.0021 & 0.0004 & 0.0001 & 0.0000 \\
\hline Portfolio 29 & 113,67 & BB4 & 0.0461 & 0.0083 & 0.0020 & 0.0006 & 0.0002 \\
\hline Portfolio30 & 91,99 & Frank & 0.1157 & 0.0376 & 0.0121 & 0.0046 & 0.0020 \\
\hline Portfolio 31 & 34,103 & BB1 & 0.0999 & 0.0257 & 0.0086 & 0.0032 & 0.0013 \\
\hline Portfolio32 & 18,11 & Normal & 0.1160 & 0.0449 & 0.0174 & 0.0065 & 0.0026 \\
\hline Portfolio33 & 39,55 & BB1 & 0.0571 & 0.0071 & 0.0016 & 0.0005 & 0.0002 \\
\hline Portfolio 34 & 102,69 & BB1 & 0.0516 & 0.0089 & 0.0021 & 0.0006 & 0.0002 \\
\hline Portfolio $_{35}$ & 37,44 & BB1 & 0.1626 & 0.0708 & 0.0294 & 0.0130 & 0.0062 \\
\hline
\end{tabular}

Table 6: Probability of Simultaneous Percentage losses

This table presents the probability that both assets in the portfolio decline by the same amounts or more. A value of 0.1 in the table corresponds to 10 per cent. Asset numbers and the corresponding tickers are presented in Table 9 . 


\begin{tabular}{|c|c|c|c|c|c|c|}
\hline \multicolumn{7}{|c|}{ PORTFOLIOS OF BONDS } \\
\hline & \multicolumn{6}{|c|}{ Simultaneous Losses } \\
\hline & Copula & 1 per cent & 2 per cent & 3 per cent & 4 per cent & 5 per cent \\
\hline Portfolio 1 & BB4 & 0.0041 & 0.0001 & 0.0000 & 0.0000 & 0.0000 \\
\hline Portfolio $_{2}$ & BB4 & 0.0016 & 0.0002 & 0.0000 & 0.0000 & 0.0000 \\
\hline Portfolio3 & BB1 & 0.0060 & 0.0004 & 0.0000 & 0.0000 & 0.0000 \\
\hline Portfolio $_{4}$ & BB1 & 0.0055 & 0.0001 & 0.0000 & 0.0000 & 0.0000 \\
\hline Portfolio 5 & BB1 & 0.0104 & 0.0004 & 0.0000 & 0.0000 & 0.0000 \\
\hline Portfolio $_{6}$ & BB1 & 0.0073 & 0.0001 & 0.0000 & 0.0000 & 0.0000 \\
\hline Portfolio7 & BB4 & 0.0001 & 0.0000 & 0.0000 & 0.0000 & 0.0000 \\
\hline Portfolio $_{8}$ & BB4 & 0.0014 & 0.0001 & 0.0000 & 0.0000 & 0.0000 \\
\hline Portfoliog & Frank & 0.0000 & 0.0000 & 0.0000 & 0.0000 & 0.0000 \\
\hline Portfolio10 & BB1 & 0.0066 & 0.0000 & 0.0000 & 0.0000 & 0.0000 \\
\hline Portfolio $11_{1}$ & BB1 & 0.0040 & 0.0000 & 0.0000 & 0.0000 & 0.0000 \\
\hline Portfolio $_{12}$ & BB4 & 0.0053 & 0.0002 & 0.0000 & 0.0000 & 0.0000 \\
\hline Portfolio 13 & BB1 & 0.0064 & 0.0002 & 0.0000 & 0.0000 & 0.0000 \\
\hline Portfolio14 & BB1 & 0.0062 & 0.0000 & 0.0000 & 0.0000 & 0.0000 \\
\hline Portfolio $_{15}$ & BB1 & 0.0011 & 0.0001 & 0.0000 & 0.0000 & 0.0000 \\
\hline Portfolio 16 & BB1 & 0.0120 & 0.0003 & 0.0000 & 0.0000 & 0.0000 \\
\hline Portfolio 17 & BB1 & 0.0036 & 0.0001 & 0.0000 & 0.0000 & 0.0000 \\
\hline Portfolio18 & BB1 & 0.0067 & 0.0001 & 0.0000 & 0.0000 & 0.0000 \\
\hline Portfolio $19_{19}$ & BB1 & 0.0069 & 0.0004 & 0.0000 & 0.0000 & 0.0000 \\
\hline Portfolio $_{20}$ & BB1 & 0.0032 & 0.0001 & 0.0000 & 0.0000 & 0.0000 \\
\hline Portfolio $_{21}$ & BB1 & 0.0073 & 0.0001 & 0.0000 & 0.0000 & 0.0000 \\
\hline Portfolio $_{22}$ & BB4 & 0.0001 & 0.0000 & 0.0000 & 0.0000 & 0.0000 \\
\hline Portfolio $_{23}$ & BB4 & 0.0014 & 0.0001 & 0.0000 & 0.0000 & 0.0000 \\
\hline Portfolio $_{24}$ & Frank & 0.0000 & 0.0000 & 0.0000 & 0.0000 & 0.0000 \\
\hline Portfolio 25 & BB1 & 0.0066 & 0.0000 & 0.0000 & 0.0000 & 0.0000 \\
\hline
\end{tabular}

Table 7: Probability of Simultaneous PERCENTAGE LOSSES

This table presents the probability that both assets in a bond portfolio decline by the same amounts or more. A value of 0.1 in the table corresponds to 10 per cent. Bonds included in this table have a maturity of 10 years or more.

Another way of determining the portfolios with the lowest probability of simultaneous losses is to construct a risk-cost frontier from Tables 6 and 7 in a similar way as Garcia and Gençay (2006). For this application of the frontier, risk corresponds to the probability of simultaneous losses, and the cost corresponds to the actual percentage losses for each asset. In Figures 8 and 9 we present selected risk-cost frontiers for groups of five equity and bond portfolios. Figure 8 shows the frontier for ten portfolios of equities. The first five are shown in the upper panel and the remaining in the lower panel. In both panels, the $x$-axis corresponds to the percentage losses for both assets of the portfolio, and the $y$-axis represents the probability of observing simultaneous losses in both assets. Each curve represents one portfolio. Similarly, Figure 9 illustrates the frontiers for portfolios of bonds. For both of these figures the risk-cost frontiers that are further away from the origin $(x-y$-coordinates 0,0$)$ correspond to those portfolios with greater probability of simultaneous losses. In the case of bonds, the frontier constructed from Table 6 permits the easy identification of those portfolios which should be preferred from the others because they exhibit a lower joint probability of losses. A noticeable difference with equity portfolios is that as the simultaneous losses increase, bond portfolios converge to probabilities close to zero much faster than equities. 

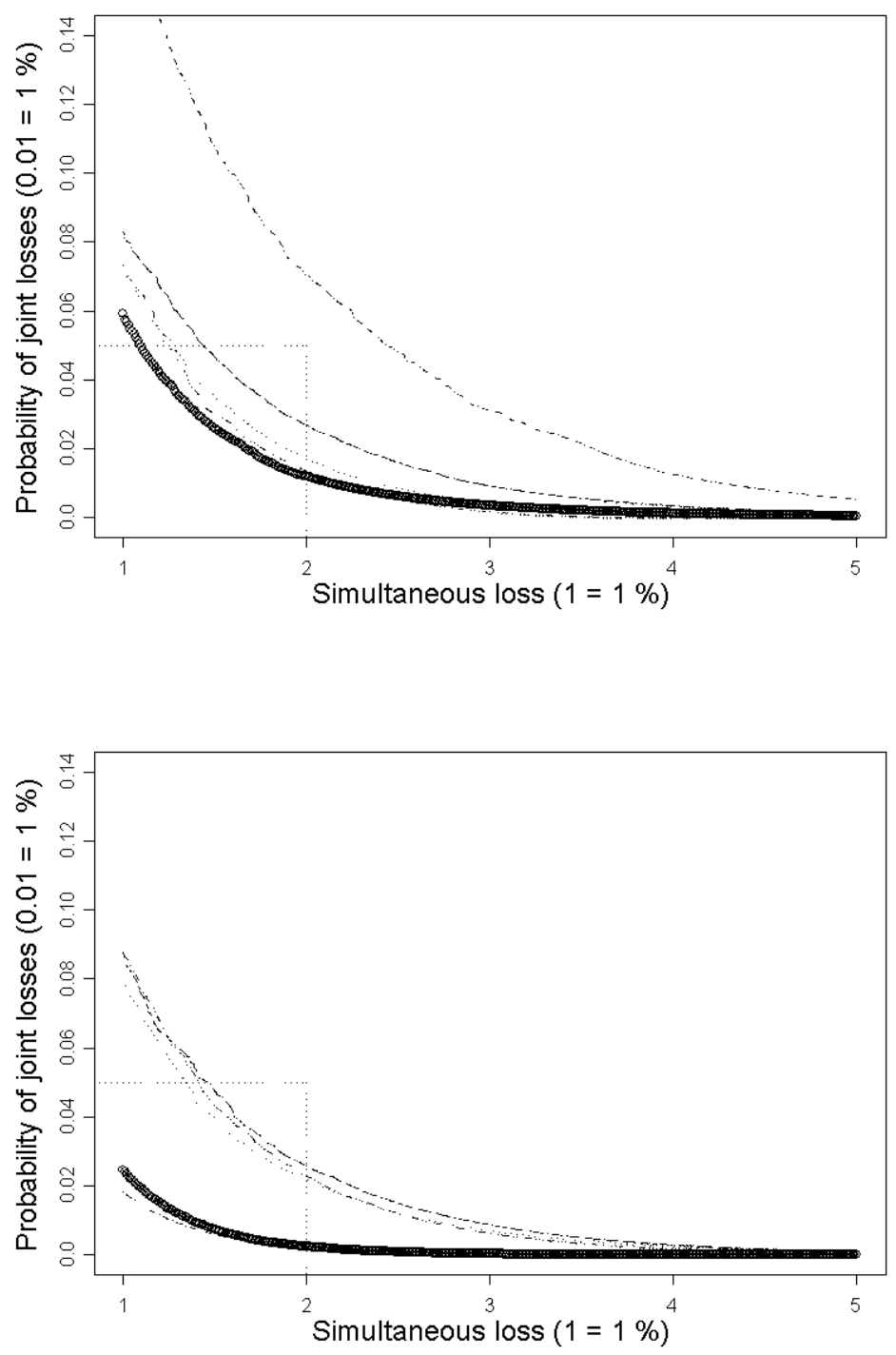

Figure 8: Risk-Cost Frontier for Equity Portfolios

This figure illustrates the risk-cost frontier for ten portfolios of equity instruments. The first five are shown in the upper panel and the remaining in the lower panel. In both panels, the $x$-axis corresponds to the percentage losses for both assets of the portfolio, and the $y$-axis represents the probability of observing simultaneous losses in both assets. Each curve represents one portfolio. Dotted horizontal and vertical lines represent threshold levels necessary for strategy 3. Threshold levels correspond to: 5 per cent probability, and 2 per cent simultaneous losses. 

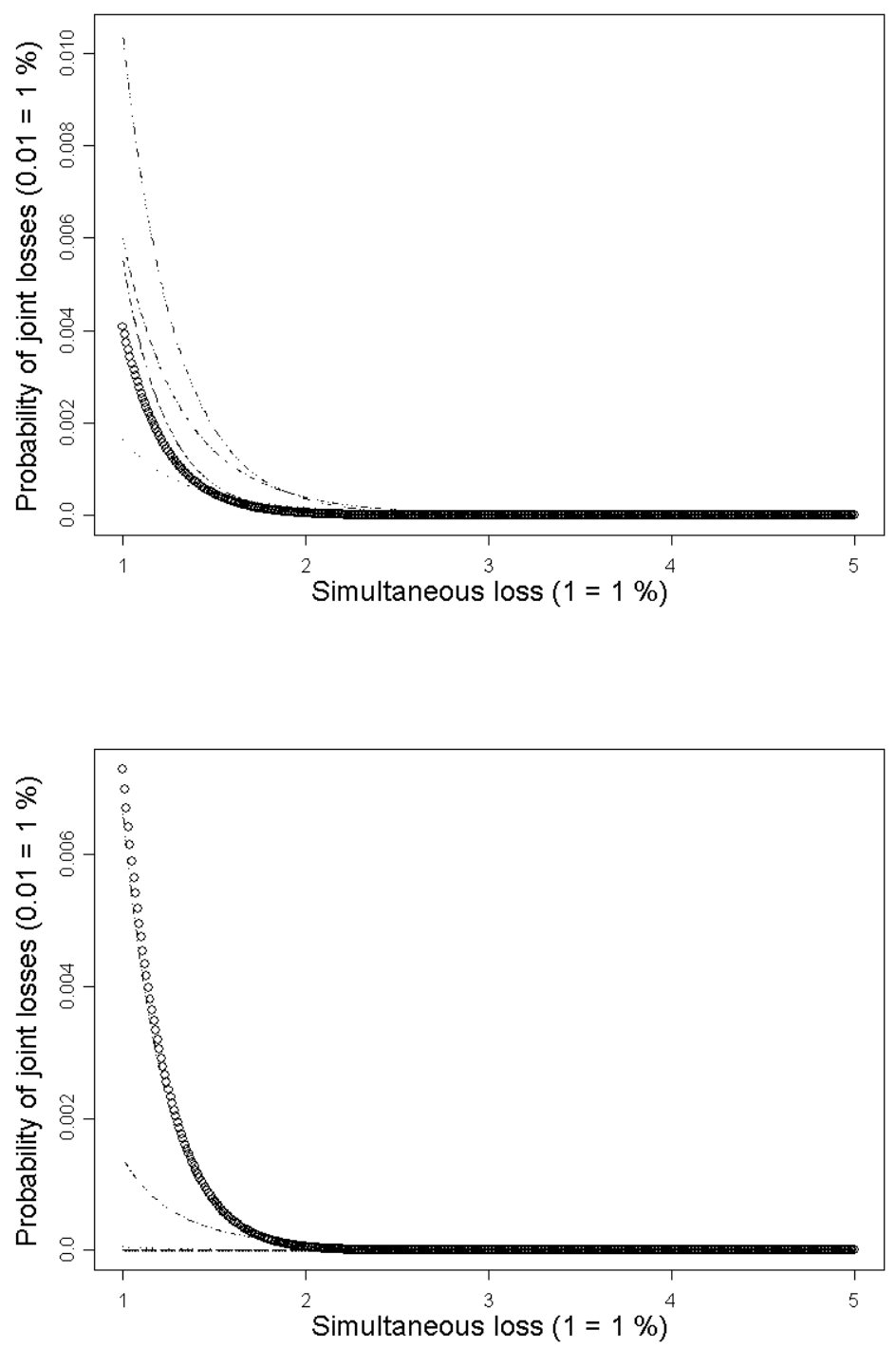

Figure 9: Risk-Cost Frontier for Bond Portfolios

This figure illustrates the risk-cost frontier for the first ten portfolios of bond securities with maturities of 10 years or more. The first five are shown in the upper panel and the remaining in the lower panel. In both panels, the $x$-axis corresponds to the percentage losses for both assets of the portfolio, and the $y$-axis represents the probability of observing simultaneous losses in both assets. Each curve represents one portfolio. 


\section{Strategy 2: Set threshold values for the probability of losses and the magnitude of the associated losses}

This strategy requires the selection of a threshold for (i) the probability associated with having simultaneous losses in both assets of the portfolio, and (ii) the magnitude of those losses. Setting the threshold levels is a function of the capital the organization has set aside to cover extreme events.

Figure 8 shows an example where the thresholds are: 5 per cent for the probability of simultaneous losses, and 2 per cent for the simultaneous losses (dotted lines denote the interception of threshold levels). Using the thresholds (dotted lines), a risk manager could re-balance or replace those portfolios that exceed the threshold levels. For example, in the upper panel of Figure 8 we observe that one portfolio exceeds the thresholds, therefore the risk manager following Strategy 3 may replace this portfolio with others which are below the threshold levels.

\section{Strategy 3: Stress test the dependence structure for the portfolio of col- lateral}

This strategy is meant to address the question: How would the portfolio perform if there was an event that negatively affected the historical dependence structure? To answer this question we use portfolio 1 to illustrate a stress test of the dependence structure. The stress test consists of changing the dependence structure for the marginal return distributions. This requires us to consider what would be the appropriate copula that can be used as the extreme dependence case? We propose (i) testing using a comprehensive set of copula families, and (ii) increasing the degree of positive dependence for each copula. This approach will provide an envelop of the possible adverse dependence that may exist during 'systemic events'. During such events, situations arise where the supply of securities exceeds the demand, driving down the market value. Stress testing the dependence could provide information about the magnitude of losses when events of this nature occur.

To stress test the dependence structure, we increase the level of positive dependence, using as a starting value the pairwise historical correlation. To illustrate this methodology, we use portfolio 1 . The historical correlation for portfolio 1 is 0.68 . To stress test the dependence, we increase the correlation (up to 0.99) maintaining the characteristics of the marginals. For the different correlations we estimate the 99 per cent Value-at-Risk for the equally weighted portfolio and report the results in Table 8. We report the results for three models of the joint return distribution: the first model is the one with t-marginals and a Gumbel copula; the second model is the one with t-marginals and a normal copula; and the third model is the one with normal marginals and a normal copula. The last one can be thought of as simply a bivariate normal distribution. We compare the three models to show how different the resulting losses can be. As can be observed in the table, the bivariate normal model consistently gives losses that are lower than those reported by the copula models. Although it is not known what the dependence structure will be during 
stress episodes, the copula approach to model dependence permits one to construct plausible scenarios. The results presented in Table 8 are only meant to highlight the importance of choosing various models for the joint distribution to stress test the dependence, since the actual outcomes may be quite different.

\begin{tabular}{lccccccc}
\hline & \multicolumn{7}{c}{ CORRELATION } \\
VaR & 0.68 & 0.70 & 0.75 & 0.80 & 0.85 & 0.95 & 0.99 \\
\hline Gumbel Copula & 4.1182 & 4.1643 & 4.1987 & 4.2461 & 4.2967 & 4.3688 & 4.3825 \\
Standard Error & $(0.0019)$ & $(0.0019)$ & $(0.0020)$ & $(0.0023)$ & $(0.0021)$ & $(0.0020)$ & $(0.0024)$ \\
Normal Copula & 3.9518 & 4.0028 & 4.0637 & 4.1139 & 4.2224 & 4.3157 & 4.4235 \\
Standard Error & $(0.0019)$ & $(0.0018)$ & $(0.0020)$ & $(0.0020)$ & $(0.0021)$ & $(0.0024)$ & $(0.0022)$ \\
Bivariate Normal & 3.5246 & 3.5591 & 3.6104 & 3.6545 & 3.7216 & 3.8054 & 3.8675 \\
Standard Error & $(0.0007)$ & $(0.0008)$ & $(0.0009)$ & $(0.0008)$ & $(0.0009)$ & $(0.0010)$ & $(0.0008)$ \\
\hline
\end{tabular}

Table 8: Portfolio Losses as Correlation Increases

This table presents the losses for portfolio 1 using a 99 per cent Value-at-Risk measure on the empirical distribution for different levels of positive dependence. We use three models to capture the dependence: Normal copula with semi-parametric GPD marginals; Gumbel copula also with semi-parametric GPD marginals; and a bivariate normal distribution. We conduct a Monte Carlo simulation with 100 repetitions and report the average VaR at the 99 confidence level and the corresponding standard error. A value of 1 in the table corresponds to 1 per cent loss. Losses are represented with positive values.

\section{Conclusions}

In this paper we propose various strategies to manage the market risk of portfolios of securities. These strategies are designed to help reduce the losses that may arise during normal and extreme market events. In proposing these strategies we highlight that it is important to include in the risk management framework scenarios that not only account for the realization of low quantiles in the return distributions of individual assets, but also account for adverse changes in the dependence structure of the portfolio (e.g., a structure that exhibits lower or no diversification benefits). These strategies are presented in a flexible framework that permits one to model separately the marginal distributions, and the dependence structure. This is achieved by adopting a copula approach. These strategies include (i) a framework to calculate haircuts for individual assets, (ii) a methodology to monitor changes in the dependence structure during normal times, and (iii) a methodology to stress test and measure the possible effects on the portfolio value of adverse dependence structures. The proposed risk management strategies contribute to the existing policies to measure and manage market risk during episodes where the dependence structure becomes highly positively correlated.

There is at least one possible direction for future research. This is to study the stylized facts of the dependence structure during extreme events with the objective of recommending the copula that may provide the most accurate characterization. 


\section{References}

Balkema, A. A. and de Haan, L. (1974). Residual lifetime at great age. Annals of Probability, 2, 792-804.

BIS (2001a). Core principals for systemically important payment systems. Bank for International Settlements and International Organization of Securities Commissions Report.

BIS (2001b). Recommendations for securities settlement systems. Bank for International Settlements and International Organization of Securities Commissions Report.

BIS (2004). Recommendations for central counterparties. Bank for International Settlements and International Organization of Securities Commissions Report.

Carmona, R. (2004). Statistical Anlaysis of Financial Data in Splus. Springer.

Chan, N., Getmansky, M., Haas, S., and Lo, A. (2005). Systemic risk and hedge funds. MIT Sloan School of Management working paper.

Clayton, D. (1978). A model for association in bivariate life tables and its application in epidemiological studies of familial tendency in chronic disease incidence. Biometrika, 65, 144-51.

Durrleman, V., Nikeghbali, A., Riboulet, G., and Roncalli, T. (2000). Copulas for finance a reading guide and some applications. unpublished.

Embrechts, P., Lindskog, F., and McNeil, A. (2001). Modeling dependence with copulas and applications to risk management. Mimeo Department of Mathematics ETHZ.

Garcia, A. and Gençay, R. (2006). Risk-cost frontier and collateral valuation in securities settlement systems for extreme market movements. Bank of Canada Working Paper 19.

Kimeldorf, G. and Sampson, A. (1975). Uniform representations of bivariate distributions. Communications in Statistics, 4, 617-27.

Pickands, J. (1975). Statistical inference using extreme order statistics. Annals of Statistics, 3, 119-131.

Schmidt, T. (2006). Coping with copulas. Chapter forthcoming in Risk Books: Copulas from theory to applications in finance.

Sklar, A. (1959). Fonctions de répartition à n dimensions et leurs marges. Publications de l'Institut de Statistique de l'Université de Paris.

Zivot, E. and Wang, J. (2006). Modeling Financial Time Series with Splus. Springer. 


\section{Appendix}

\begin{tabular}{|c|c|c|c|c|c|c|c|}
\hline Asset & Ticker & Asset & Ticker & Asset & Ticker & Asset & Ticker \\
\hline 2 & RY.TO & 41 & IGI.TO & 80 & FDG.u.TO & 119 & RON.TO \\
\hline 3 & BNS.TO & 42 & ERF.u.TO & 81 & SPF.u.TO & 120 & MX.TO \\
\hline 4 & TD.TO & 43 & RCIb.TO & 82 & MRUa.TO & 121 & TIH.TO \\
\hline 5 & BMO.TO & 44 & A.TO & 83 & ABZ.TO & 122 & PEY.u.TO \\
\hline 6 & ВСЕ.TO & 45 & WN.TO & 84 & MHM.TO & 123 & IPL.u.TO \\
\hline 7 & CM.TO & 46 & CIX.TO & 85 & Х.TO & 124 & DIIb.TO \\
\hline 8 & IMO.TO & 47 & РОТ.TO & 86 & PKZ.TO & 125 & CGSs.TO \\
\hline 9 & ECA.TO & 48 & CIX.TO & 87 & ATDb.TO & 126 & SMU.u.TO \\
\hline 10 & AL.TO & 49 & COS.u.TO & 88 & CSN.TO & 127 & ALRb.TO \\
\hline 11 & NT.TO & 50 & ATY.TO & 89 & OCX.TO & 128 & ACOx.TO \\
\hline 12 & SU.TO & 51 & CTRa.TO & 90 & NMC.TO & 129 & ROC.TO \\
\hline 13 & MFC.TO & 52 & HSE.TO & 91 & GIBa.TO & 130 & IMG.TO \\
\hline 14 & SLF.TO & 53 & TA.TO & 92 & TCLa.TO & 131 & PTF.u.TO \\
\hline 15 & PCA.TO & 54 & SJRb.TO & 93 & TEU.TO & & \\
\hline 16 & TRP.TO & 55 & BPO.TO & 94 & WRM.TO & & \\
\hline 17 & CNQ.TO & 56 & Т.TO & 95 & PJCa.TO & & \\
\hline 18 & TLM.TO & 57 & MDS.TO & 96 & GLG.TO & & \\
\hline 19 & BBDb.TO & 58 & REI.u.TO & 97 & TPL.u.TO & & \\
\hline 20 & CNR.TO & 59 & PWT.TO & 98 & WTO.TO & & \\
\hline 21 & ENB.TO & 60 & NCX.TO & 99 & WJA.TO & & \\
\hline 22 & POW.TO & 61 & DTC.TO & 100 & BVF.TO & & \\
\hline 23 & ABX.TO & 62 & AET.u.TO & 101 & HR.u.TO & & \\
\hline 24 & MGa.TO & 63 & G.TO & 102 & IAG.TO & & \\
\hline 25 & NA.TO & 64 & YLO.u.TO & 103 & CRW.TO & & \\
\hline 26 & BNNa.TO & 65 & MOLa.TO & 104 & CU.TO & & \\
\hline 27 & TOC.TO & 66 & DFS.TO & 105 & AGU.TO & & \\
\hline 28 & L.TO & 67 & FL.TO & 106 & AGFb.TO & & \\
\hline 29 & NRD.TO & 68 & PD.TO & 107 & QLT.TO & & \\
\hline 30 & PWF.TO & 69 & МВТ.ТО & 108 & AIT.TO & & \\
\hline 31 & NXY.TO & 70 & SNC.TO & 109 & ACMa.TO & & \\
\hline 32 & RIM.TO & 71 & FTT.TO & 110 & PIF.u.TO & & \\
\hline 33 & PDG.TO & 72 & TER.TO & 111 & BCB.TO & & \\
\hline 34 & XIU.TO & 73 & K.TO & 112 & CAE.TO & & \\
\hline 35 & GWO.TO & 74 & PGF.u.TO & 113 & SIF.u.TO & & \\
\hline 36 & SC.TO & 75 & IQW.TO & 114 & UP.TO & & \\
\hline 37 & TEKb.TO & 76 & CLS.TO & 115 & FHR.TO & & \\
\hline 38 & N.TO & 77 & NF.TO & 116 & SAP.TO & & \\
\hline 39 & CP.TO & 78 & EMA.TO & 117 & ESI.TO & & \\
\hline 40 & SHC.TO & 79 & FFH.TO & 118 & OTC.TO & & \\
\hline
\end{tabular}

Table 9: EquiTy DATA

This table shows the ticker and the corresponding number for all the equity instruments in the database. 